\title{
Técnica de Medição de Vazão por Vertedor Triangular de Parede Delgada, Instalado na UFMT
}

\section{Technical Flow Measurement in Spillway Thin Wall Triangular, Installed on UFMT}

\author{
Bruno Leonel Rossi ${ }^{1}$ (bruno_rossi_@ hotmail.com) \\ Karen Rebeschini de Lima²(karen_rebeschini@ ${ }^{2}$ hotmail.com) \\ Katia Hermann ${ }^{3}$ (katiahermann_13@ hotmail.com) \\ Keyla Rodrigues Santa Catharina ${ }^{4}$ (keyla_rsc@hotmail.com) \\ ${ }^{1}$ Engenheiro Sanitarista e Ambiental, Cuiabá MT. \\ ${ }^{234}$ Engenheira Sanitarista e Ambiental, Acadêmica Engenharia de Segurança do Trabalho, Cuiabá MT.
}

\begin{abstract}
RESUMO
O presente trabalho teve como objetivo medir a vazão deste canal, analisar o comportamento do mesmo durante o período de estudo e verificar a adequabilidade da metodologia utilizada quanto ao comportamento da vazão. A área de estudo, localiza-se no canal do Departamento de Engenharia Sanitária e Ambiental que faz parte do bloco da Faculdade de Engenharia e Tecnologia da Universidade Federal de Mato Grosso - UFMT. Para isso, utilizou-se o vertedor triangular de parede delgada, e mediu-se a altura da carga hidráulica com uma régua durante o período de 5 (cinco) dias. E, por meio da equação de Gourley e Crimp obteve-se o valor da vazão instantânea igual a $0,23 \mathrm{~L} / \mathrm{s}$.Após o período de medição em campo, fez-se uma simulação hipotética de diferentes alturas de laminas d'água no canal, a fim de comparar a relação entre a altura da lâmina d'água no vertedor (h) e que altura essa lâmina d'água representaria no canal (y), e verificou-se que:quando o vertedor atingir uma altura de $0,030 \mathrm{~m}$, esta altura representaria no canal $0,004 \mathrm{~m}$ em condições normais.
\end{abstract}

Palavras-Chave: Canal FAET, Determinação de Vazão, Equação de Gourley e Crimp.

\begin{abstract}
This study aimed to measure the Flow of this channel, analyze the same behavior during the study period and verify the appropriate ness of the methodology used regarding the behavior of the flow. The study area, located in the Department of Sanitary and Environmental Engineering of the channel which is part of the block of the Faculty of Engineering and Technology of the Federal University of Mato Grosso - UFMT. For this, we used the triangular spout thinwall, and measured the height of the hydraulic head with a slit for the period of five (5) days. And, by Gourley and Crimp equation obtained if the value of the instantaneous flow rate equal to $0.231 /$ s. After the measurement period in the field, there was a hypothetical simulating blades of different heights in water channel, to compare there lation ship between the height of the weir water depth (h) and that this time would represent water depth in the channel (y), and it was found that: when the spout reach a height of $0.030 \mathrm{~m}$, this time represent the channel $0.004 \mathrm{~m}$ under normal conditions.
\end{abstract}

Keywords: Channel FAET, Flow Determination, Gourley and Crimp Equation.

\section{INTRODUÇÃO}

O gerenciamento dos recursos hídricos em bacias hidrográficas é de suma importância devido ao cenário de degradação das águas e à necessidade de novas fontes de abastecimento, bem como para conhecer os regimes fluviométricos das bacias, $\mathrm{o}$ comportamento dos rios e suas vazões.
Dessa forma, a medição de vazão é uma ferramenta fundamental na caracterização das condições hidrológicas e na disponibilidade hídrica de certa bacia. Existem diversas maneiras de se obter a vazão, a escolha do método depende do volume do fluxo de água, das condições locais, do custo e da precisão desejada. Dentre os 
métodos mais simplificados, encontramse os vertedores, que são aberturas na parte superior de uma parede, onde o líquido escoa, e são indicados para medir de pequenas a médias vazões. Geralmente são construídos com forma geométrica definida, sendo as mais utilizadas: retangulares, triangulares, trapezoidais e circulares.

Optou-se por utilizar o vertedor triangular de parede delgada, pois segundo a NBR 13403/95 os vertedores triangulares oferecem maior precisão para vazões menores que $30 \mathrm{~L} / \mathrm{s}$ - sendo esta uma característica do canal em estudo - são fáceis de instalar, e as determinações contínuas de vazão são possíveis quando um registrador de altura é acoplado ao vertedor.

O presente trabalho tem como objetivo medir a vazão do canal artificial localizado nas proximidades Figura 1 - Localização do canal DESA/FAET e do ponto de instalação do vertedor.

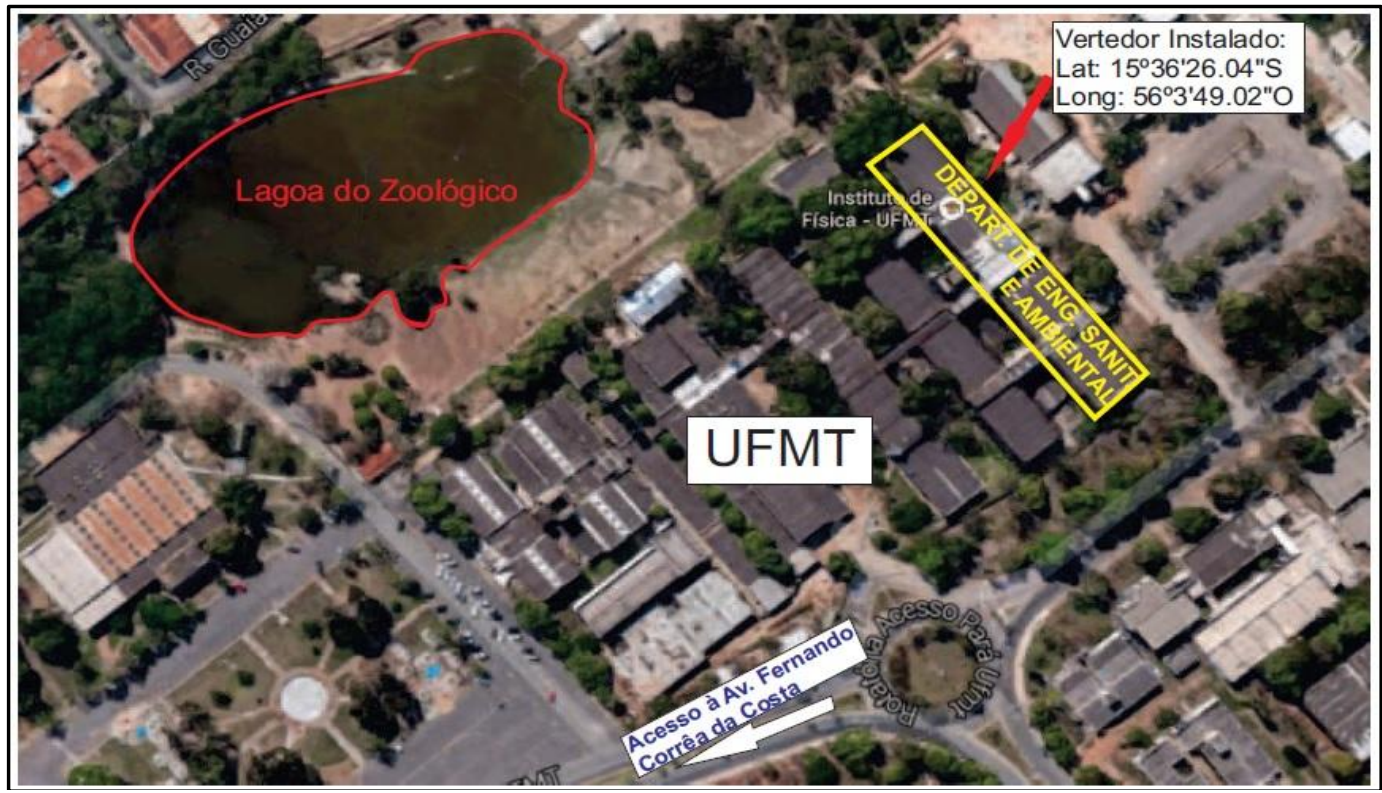

Fonte: Google Earth

\section{METODOLOGIA}

Caracterização do canal e escolha do método utilizado

Inicialmente realizou-se uma caracterização do canal em estudo através de observações visuais, medição do Departamento de Engenharia Sanitária e Ambiental (DESA), da Universidade Federal do Mato Grosso (UFMT), e verificar a adequabilidade da metodologia utilizada quanto ao comportamento da vazão, tendo em vista os princípios da hidráulica em condutos livres.

\section{Área de estudo}

O canal localiza-se aos fundos do DESA, na Faculdade de Arquitetura, Engenharia e Tecnologia(FAET), da UFMT. Este recebe contribuições da nascente localizada no fundo do departamento, do Instituto de Saúde Coletiva e dos laboratórios instalados no departamento.O local escolhido para instalar o vertedor está entre a latitude $15^{\circ} 36^{\prime} 26,04^{\prime \prime} \mathrm{S}$ e a longitude $56^{\circ} 3$ '49,02'O, como mostra a Figura 1. 
projetou-se um vertedor de madeirite (12 $\mathrm{mm}$ de espessura), com as seguintes características: forma geométrica triangular com abertura de $90^{\circ} \mathrm{e}$ contração lateral, ângulo interno nas laterais igual a $115^{\circ}$, base menor de $0,98 \mathrm{~m}$ e altura de $0,50 \mathrm{~m}$, sendo $0,20 \mathrm{~m}$ a altura da superfície até o vértice do triangulo (Figura 2).

Figura 2 - Vertedor triangular

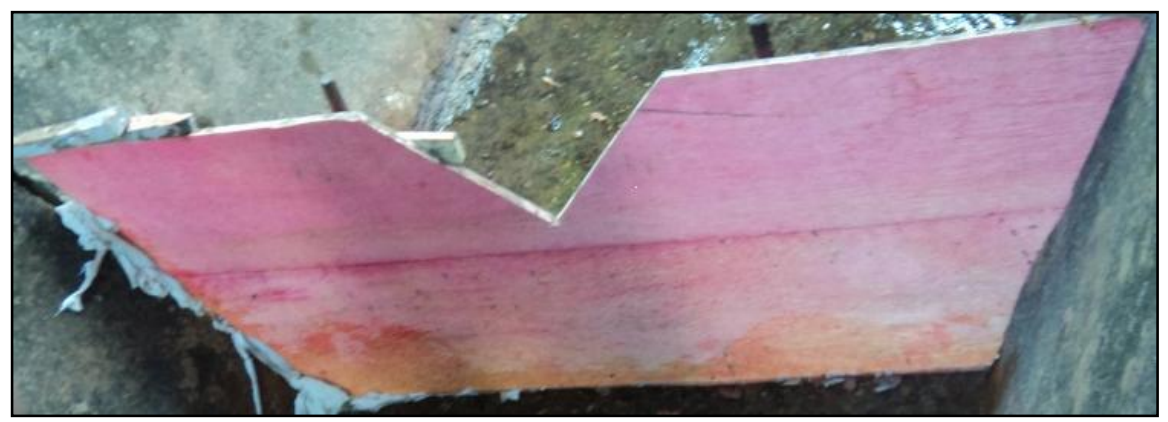

Instalação do vertedor e medição carga hidráulica

$\mathrm{O}$ vertedor foi apoiado no fundo e nas laterais do canal. Em seguida cravou-se 3 (três) estacas de ferro a jusante do vertedor, para garantir a verticalidade da placa, ou seja, evitar que a força da água provoque um empenamento do mesmo. Além disso, cravou-se tocos de madeira na parte superior do mesmo de forma a evitar seu deslocamento e colocou-se estopa a fim de vedar a passagem da água na lateral e no fundo do vertedor (Figura $3)$.

Figura 3 - Posicionamento das estacas de ferro e dos tocos de madeira

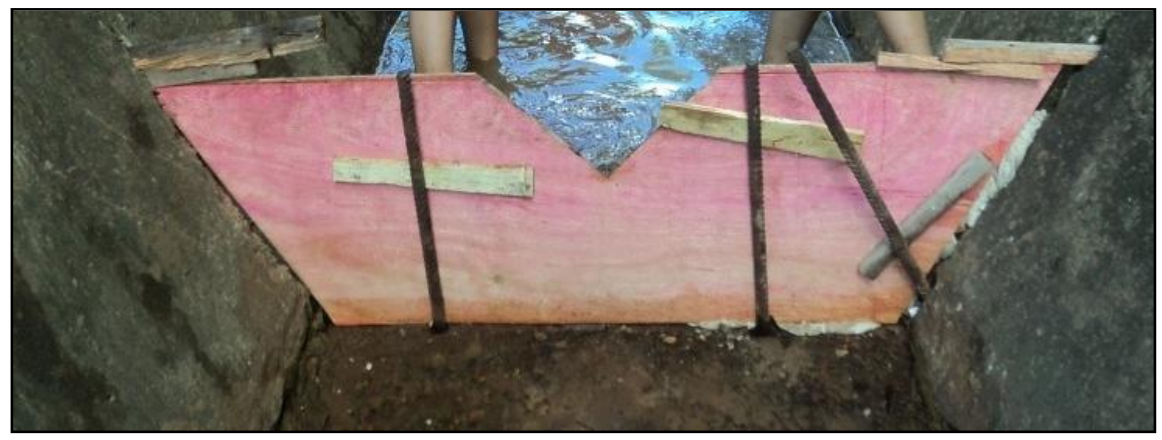

Posteriormente, com o vertedor já fixado no canal, adicionou-se argila a montante do mesmo e compactou-a (Figura 4-a), a fim de garantir a estanqueidade da água pelas laterais e pela parte inferior do vertedor (Figura 4-b). 
Figura 4 - (a)Compactação da argila;

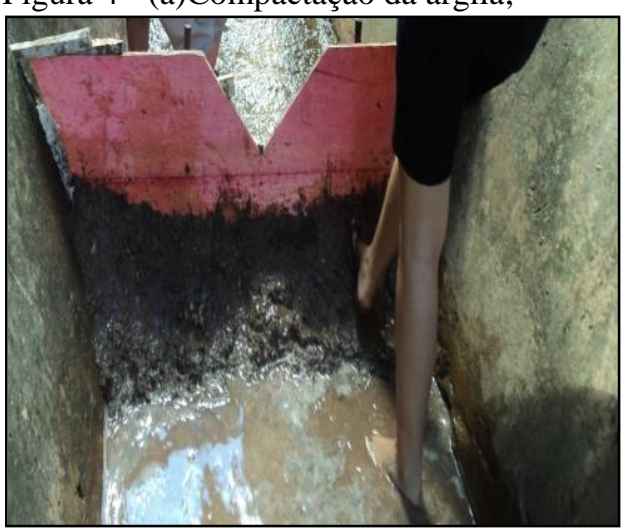

$\mathrm{O}$ vertedor foi instalado no dia 22 de abril de 2012 e a primeira medição foi realizada no dia seguinte, às 07:00 horas.

A medição da carga hidráulica (h) foi feita a partir de uma régua acima da soleira (Figura 5), em média, 3 (três) vezes ao dia, no período de 5 (cinco) dias. Sendo a primeira medição realizada 15 horas após a instalação do vertedor

Figura 5 - Medição da carga hidráulica

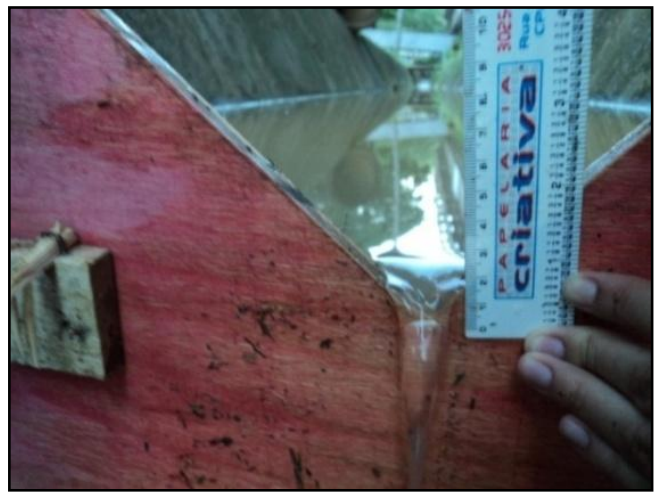

A equação utilizada para determinar a vazão no vertedor foi aequação de Gourley e Crimp, dada por (Equação 1).

$\mathbf{Q}=\mathbf{1}, 32 \times \mathbf{h}^{2,47}$

Equação 1

Onde: $\mathrm{h}=$ carga hidráulica $(\mathrm{m})$.

Método Iterativo e classificação do escoamento
Figura 4 - (b) Estanqueidade da água devido adição da argila.

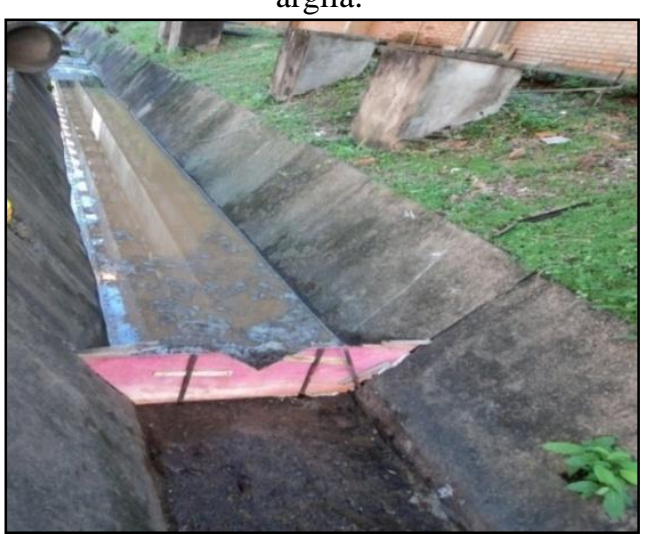

Após o período de medição em campo, fez-se uma simulação hipotética de diferentes alturas de laminas d'água no canal, a fim de comparar a relação entre a altura da lâmina d'água no vertedor (h) e a lâmina d'água no canal (y). Para isso utilizou-se o método iterativo e calculou-se as respectivas vazões no canal às alturas de laminas d'água (y) atribuídas, através da equação de Manning (Equação 2).

$$
Q=\frac{1}{n} \times A \times R_{h}^{2 / 3} \times I_{o}^{1 / 2} \text { Equação } 2
$$

Onde:

$\mathrm{n}=$ coeficiente de rugosidade de Manning, igual a 0,013;

$\mathrm{A}=$ área molhada $\left(\mathrm{m}^{2}\right)$;

$\mathrm{R}_{\mathrm{h}}=$ raio hidráulico $(\mathrm{m})$, dado pela relação entre a área molhada e o perímetro molhado;

$\mathrm{I}_{\mathrm{o}}=$ declividade do canal $(\mathrm{m} / \mathrm{m})$.

A partir disso calculou-se a velocidade do escoamento no canal por meio da equação de Manning (Equação3).

$$
V=\frac{1}{n} \times R_{h}^{2 / 3} \times I_{o}^{1 / 2} \text { Equação } 3
$$

Em seguida, calculou-se o número de Froude $\left(\mathrm{F}_{\mathrm{r}}\right)$ a fim de caracterizar o escoamento do canal (Equação 4). 
Rossi B. L., Lima K. R., Hermann K., Catharina K. R. S., Técnica de Medição de Vazão por Vertedor Triangular de Parede Delgada, Instalado na UFMT. E\&S - Engineering and Science 2015, 1:3

Onde:

$$
\boldsymbol{F}_{\boldsymbol{r}}=\frac{\boldsymbol{V}}{\sqrt{\boldsymbol{g} \times \boldsymbol{y}}} \text { Equação } 4
$$

$\mathrm{V}=$ velocidade do escoamento $(\mathrm{m} / \mathrm{s})$;

$\mathrm{g}=$ aceleração da gravidade, igual 9,81;

$\mathrm{y}=$ altura da lâmina d'água no canal.

Por fim, calculou-se o número de Reynolds para classificar o regime de escoamento do fluido (Equação 5).

Onde:

$$
\boldsymbol{R}_{\boldsymbol{e}}=\frac{\boldsymbol{V} \times \boldsymbol{R}_{\boldsymbol{h}}}{v} \text { Equação } 5
$$

$\mathrm{V}=$ velocidade do escoamento $(\mathrm{m} / \mathrm{s})$;

$\mathrm{R}_{\mathrm{h}}=$ raio hidráulico;

$\mathrm{V}=$ viscosidade do fluido, igual a $1,01 \times 10^{-6}$.

Após a caracterização do escoamento e do regime no canal, utilizou-se a equação da continuidade (Equação 6) para calcular a velocidade do escoamento no vertedor e as equações 4 e 5 para caracterizar o escoamento e o regime do vertedor.

$$
\boldsymbol{V}=\frac{\boldsymbol{Q}}{\boldsymbol{A}} \quad \text { Equação } 6
$$

\begin{tabular}{|c|c|c|c|c|c|}
\hline Dia & Hora & $\mathbf{H}(\mathbf{m})$ & $\begin{array}{c}\text { Vazão diária } \\
(\mathrm{L} / \mathrm{s})\end{array}$ & $\begin{array}{c}\text { Vazão média diária } \\
(\mathrm{L} / \mathrm{s})\end{array}$ & $\begin{array}{c}\text { Coef. de variação } \\
\text { diária }(\%)\end{array}$ \\
\hline \multirow[t]{3}{*}{$23 / 4 / 2012$} & 07:00 & 0,028 & 0,190 & & \\
\hline & 13:00 & 0,030 & 0,230 & 0,280 & $42 \%$ \\
\hline & $17: 30$ & 0,038 & 0,410 & & \\
\hline \multirow[t]{3}{*}{$24 / 4 / 2012$} & 07:00 & 0,028 & 0,190 & & \\
\hline & $13: 00$ & 0,025 & 0,150 & 0,160 & $20 \%$ \\
\hline & $17: 30$ & 0,024 & 0,130 & & \\
\hline \multirow[t]{3}{*}{$25 / 4 / 2012$} & 07:00 & - & - & & \\
\hline & 13:00 & 0,030 & 0,230 & 0,230 & $0 \%$ \\
\hline & $17: 30$ & - & - & & \\
\hline \multirow[t]{3}{*}{$26 / 4 / 2012$} & 07:00 & 0,032 & 0,269 & & \\
\hline & 13:00 & 0,035 & 0,330 & 0,275 & $19 \%$ \\
\hline & $17: 30$ & 0,030 & 0,228 & & \\
\hline \multirow[t]{4}{*}{$27 / 4 / 2012$} & 07:00 & 0,035 & 0,330 & & \\
\hline & 13:00 & 0,028 & 0,190 & 0,180 & $38 \%$ \\
\hline & $17: 30$ & - & - & & \\
\hline & & & média do canal & $0,23 \mathrm{~L} / \mathrm{s}$ & \\
\hline
\end{tabular}

Tabela 1 - Carga hidráulica medida $(\mathrm{H})$ e vazões calculadas através da fórmula de Gourley e Crim.

Segundo GARCIA (1989), o coeficiente de variação é uma medida
Onde:

$\mathrm{Q}=$ vazão encontrada a partir da equação 1 ;

$\mathrm{A}=$ área molhada

\section{RESULTADOS E DISCUSSÃO}

A partir da caracterização do canal realizada, verificou-se que o canal é artificial, aberto, de formato trapezoidal, com talude na proporção $1(\mathrm{H}): 2,05(\mathrm{~V})$ e apresenta declividade igual a $1 \%$. O mesmo é construído em concreto, apresentando irregularidades no fundo em toda sua extensão.

\section{Vazão obtida no vertedor}

A instalação do vertedor no canal possibilitou a determinação indireta da vazão, através da medição do nível d'água. E, a partir dos dados obtidos nos 05 (cinco) dias de mediçãoobteve-se as vazões diárias e a vazão média do canal (L/s), como mostra a Tabela 1 . 
desvio-padrão expresso como porcentagem da média. Há uma distribuição destes valores, em que dependendo do tipo de estudo, são determinadas faixas de aceitação dos valores encontrados. Essa distribuição considera os coeficientes de variação como baixos quando inferiores a $10 \%$, médios entre 10 e $20 \%$, altos entre 20 e $30 \%$ e muito altos se superiores a $30 \%$ (GOMES, 1990). Nota-se então que a maior variação do coeficiente em relação á média advém do primeiro dia de medição, que resultou em um coeficiente de variação de $42 \%$, ou seja, um valor considerado muito alto. Porém isto pode ser explicado devido á prováveis erros na medição da carga hidráulica no vertedor ou interferência de alguma contribuição momentânea, ocasionando assim, valores acima da média registrada nos demais dias. Já o coeficiente de variação sendo aplicado nas vazões médias diárias, obteve-se

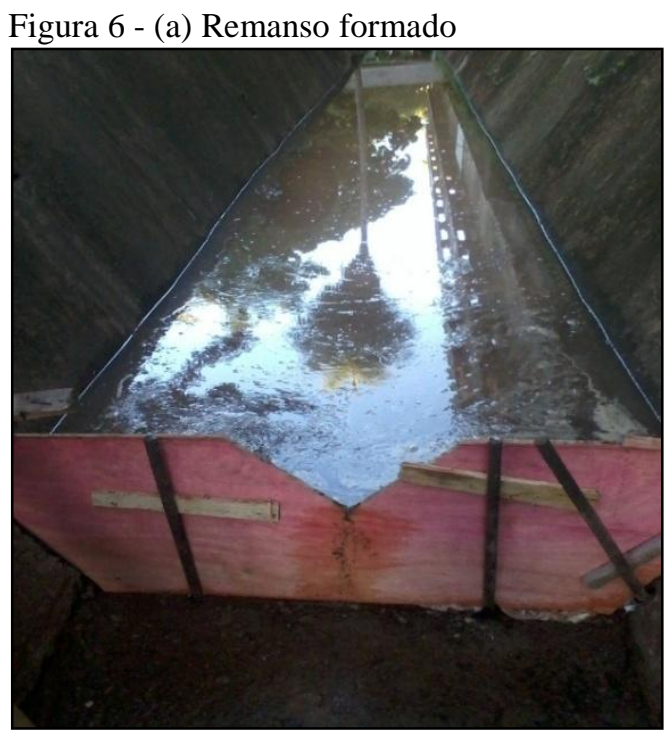

\section{Método Iterativo}

Verificou-se a partir da equação de Manning que a altura correspondente da lâmina no canal, para a vazão de $\mathrm{Q}=0,23 \mathrm{~L} / \mathrm{s}$, seria equivalente à $0,047 \mathrm{~m}$. uma variação de $27 \%$, representando assim valores altos, porém considerados dentro do nível aceitável em experimentos.

Segundo a NBR 13403/95 devese manter carga hidráulica $(\mathrm{h})$ maior que $0,05 \mathrm{~m}$, para evitar que a lâmina não goteje ao passar pelo vertedor. Como verificado na Tabela 1, mesmo não atingindo a carga hidráulica mínima recomendada, foi possível observar durante todo o período de estudo que não ocorreu gotejamento. Além disso, recomenda que seja evitada a aderência da lâmina líquida ao vertedor, e no experimento verificou-se a ocorrência de lâmina livre (Figura 6-b).

Devido a altura de $30 \mathrm{~cm}$ existente do fundo do canal até a soleira do vertedor, ocorreu um represamento da água, formando um remanso com comprimento de aproximadamente de 29,5m. (Figura 6-a).

Figura 6 - (b) Lâmina líquida vertente.

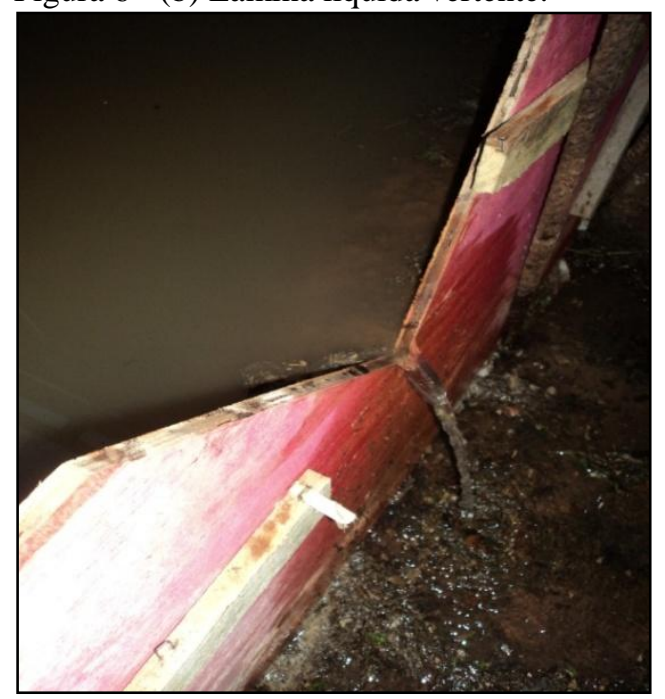

Não foi possível realizar essa medição devido às irregularidades do fundo do canal, porém a partir das medições verificou-seque a lâmina possuía em média a altura de 0,030m (Figura 7). 
Rossi B. L., Lima K. R., Hermann K., Catharina K. R. S., Técnica de Medição de Vazão por Vertedor Triangular de Parede Delgada, Instalado na UFMT. E\&S - Engineering and Science 2015, 1:3

Figura 7 - Gráfico comparativo entre as lâminas d'água no vertedor e no canal.

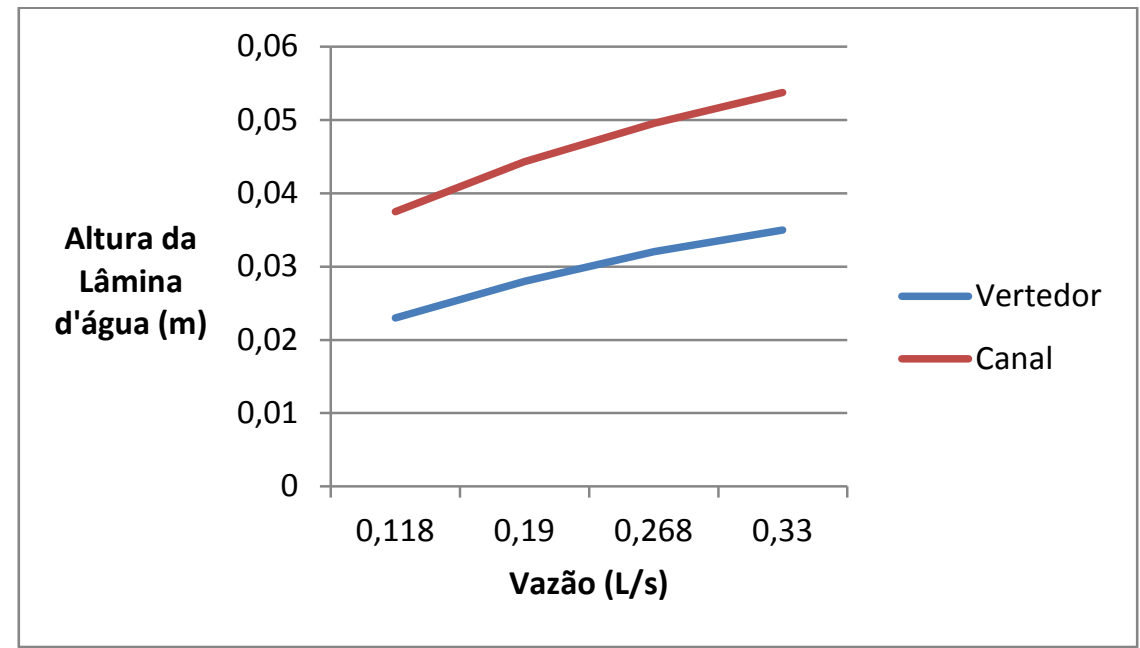

Classificação do escoamento edo regime no vertedor eno canal

Ao aplicar as equações 3, 4, 5 e 6 obteve-se os seguintes resultados - Tabela 2:

Tabela 2 - Resultados obtidos a partir da aplicação das equações 3, 4, 5 e 6

\begin{tabular}{lll}
\hline & Vertedor & Canal \\
\hline Velocidade $(\mathbf{m} / \mathbf{s})$ & 0,25 & 0,95 \\
Número de Froude & 0,46 & 1,40 \\
Número de Reynolds & 2500 & 40819,87 \\
\hline
\end{tabular}

A partir dos valores encontrados, quanto ao número de Froude, verificouse que no canal a energia cinética é preponderante sobre a energia potencial, classificando o regime em supercrítico $\left(F_{r}>1\right)$; já no vertedor ocorre o contrário, ou seja, a energia potencial é preponderante sobre a cinética, sendo, portanto, o regime subcrítico. Desta forma verifica-se que o regime passou do supercrítico para o subcrítico, fenômeno este que ocorre quando se tem o ressalto hidráulico, conforme disse CIRILLO, et, al. (2011). E, a partir da figura 6 visualiza-se o remanso formado a partir do ressalto hidráulico.

E, quanto ao número de Reynolds, verificou-se que tanto no vertedor quanto no canal, o regime é classificado como turbulento. Considerando que a equação leva em conta as forças viscosas e que o fluido que escoa é água, verifica-se que a classificação está correta, uma vez que a viscosidade (água) manteve-se igual tanto no vertedor quanto no canal.

\section{CONCLUSÃO}

Considerando que as medições foram realizadas em período relativamente pequeno, é importante ressaltar, que obteve-se a vazão instantânea do canal, uma vez que, para realizar a caracterização do comportamento hidrológico do mesmo deve-se dispor de uma série histórica de vazões, sendo mais adequado que o tempo das medições permita acompanhar os principais processos que ocorrem na bacia de drenagem, principalmente os picos de cheia que ocorrem no decorrer do ano .

Todavia vale destacar, que a simulação das alturas de lamina d'água 
realizada permitiu determinar a vazão máxima do canal o que é de grande valia, devido à crescente necessidade de informações hidrológicas em microbacias, pois estas informações podem ser utilizadas como um importante suporte no dimensionamento de alguns projetos, dentre eles o de drenagem urbana.

O método utilizado se mostrou, e constitui-se em uma alternativa segura e pratica na medição de pequenas vazões em canais abertos, Quanto aos custos deve-se considerar basicamente $\mathrm{o}$ material da placa e serviços de marcenaria para o corte da mesma, uma vez que sua instalação não exige mão de obra especializada.

Desta forma, conclui-se que a instalação do vertedor permitiu atingir os objetivos propostos, uma vez que, foi possível medir a vazão do canal.

\section{RECOMENDAÇÕES}

Recomenda-se que:

- a altura do fundo do canal até a soleira seja menor, diminuindo assim o remanso formado no canal, e consequentemente o efeito de retardo provocado pelo barramento.

- antes da instalação do vertedor seja feito testes com métodos mais simplificados - como método do flutuador -, para avaliar a vazão afluente e definir o método a ser utilizado.

- a altura da lâmina d'água no canal seja medida para que a comparação entre as vazões seja feita a partir da altura real no canal e não por iteração.

- seja utilizado o método volumétrico, como critério de aferição da vazão encontrada no vertedor.

- o vertedor seja feito de material mais resistente, evitando assim, que o mesmo seja danificado devido ao contato intenso com a água, em casos que a metodologia requerer maior período de medição.
Instalar vertedor que permita regulagem de altura para ser possível ajustes em eventos de precipitação fortes.

\section{REFERÊNCIAS BIBLIOGRÁFICAS}

ASSOCIAÇÃO BRASILEIRA DE NORMAS TÉCNICAS - ABNT - NBR 13403/1995: Medição de vazão em efluentes líquidos e corpos receptores - escoamento livre.

AZEVEDO NETTO, J. M.; ALVAREZ, G. A. Manual de Hidráulica, Ed. Edgard BlucherLtda, 8a Edição, São Paulo, 1998.

COLLISCHONN, WALTER; TASSI, RUTINÉIA. Introdução a Hidrologia. Universidade Federal do Rio Grande do Sul UFRGS, Porto Alegre, RS, 2008. Disponível em: <http://galileu. iph. ufrgs. br/collischonn/apo stila_ hidrologia/apostila.html>. Acessado em 12 de junho de 2012. (Apostila)

COSTA, FERNANDA MARTINELI; BACELLAR, LUIS DE ALMEIDA PRADO; SILVA, EDER FONSECA. Vertedores portáteis em microbacias de drenagem. Disponível em: <http://www.scielo.br/pdf/rem/ v60n2/ v60n02a02.pdf >. Acesso em 26 de junho de 2012.

GOOGLE EARTH. Guia do usuário. Disponível em: <http://earth.google.com/intl/ptBR/userguide/v4/ug_toc.html>. Acesso em: 24de junho de 2012.

HIDRÁULICA APLICADA/ Organizada por José Almir Cirillo...[et, al.] 1. Reimpressão da 2 ed. Rev. Ampl. - Porto Alegre: ABRH, 2011.

DAKER, ALBERTO. Hidráulica Aplicada à Agricultura. $1^{\mathrm{o}}$ vol. $7^{\mathrm{a}}$ ed. Rev. eAmpliação. Rio de Janeiro, RJ, 1987.

PORTO, R.M., Hidráulica Básica, 4a Ed., Escola de Engenharia de São Carlos da Universidade de São Paulo, São Carlos, 2007.

GARCIA, C.H. Tabelas para classificação do coeficiente de variação. Piracicaba: IPEF, 1989. $12 \mathrm{p}$

GOMES, F.P. O índice de variação, um substituto vantajoso do coeficiente de variação. Piracicaba: IPEF, 1991. 4p. 